It is not correct to say that "a quarter of centres did not measure height". We reported that: "Out of 42 centres, it was measured in 31, self-reported in five and not recorded whether measured or asked in six." We were being scrupulously honest and in five of the latter it is likely that height was measured. However, study personnel have moved on and definitive information could not be retrieved once we had realised that some centres had not measured height directly. In three of the centres that we classed as "self-reported height", subjects were measured if any doubt was expressed, and gross errors are unlikely to have occurred. Although the over-estimation of height in the study by STEWART et al. [3] was nontrivial, that found by NiEDHAMMER et al. [4] was $<0.5 \mathrm{~cm}$ on average. The other reference does not seem relevant.

There was exclusion for non-White ethnicity in only one centre: Melbourne (Australia). Ethnicity was not recorded in any of the other centres, which are listed in table 1 of our paper [1], but this was not raised as an issue.

It was our intention in writing the paper to engender debate and our conclusions are endorsed by S. Stanojevic and Coworkers. We believe that current reference curves cannot be guaranteed to give accurate norms of lung health, and that multicentre studies must invest substantially in standardised equipment. However, "statistical models which can adjust for between-centre differences", as advocated by S. Stanojevic and co-workers, do not solve the problem, as differences may be due to genuine variation in health.

\section{S. Chinn, D. Jarvis and P. Burney}

Imperial College, London, UK.

\section{REFERENCES}

1 Chinn S, Jarvis D, Svanes C, Burney P. Sources of variation in forced expiratory volume in one second and forced vital capacity. Eur Respir J 2006; 27: 767-773.

2 Beardsmore CS, Paton J, Thompson JR, et al. Standardized lung function laboratories for multicenter trials. Pediatr Pulmonol 2006; (In press).

3 Stewart AW, Jackson RT, Ford MA, Beaglehole R. Underestimation of relative weight by use of self-reported height and weight. Am J Epidemiol 1987; 125: 122-126.

4 Niedhammer I, Bugel I, Bonefnat S, Goldberg M, Leclerc A. Validity of self-reported weight and height in the French GAZEL cohort. Int J Obes Relat Metab Disord 2000;24: 1111-1118.

\title{
Improvement of endothelial function with allopurinol may occur in selected patients with OSA: effect of age and sex
}

\section{To the Editors:}

In a recent issue of the European Respiratory Journal, EL SOLH et al. [1] demonstrated that allopurinol improves endothelial dysfunction in patients with moderate-to-severe obstructive sleep apnoea (OSA). Because xanthine oxidase inhibition with allopurinol prevents the formation of superoxide free radicals, which leads to better endothelial function, EL SolH et al. [1] speculated that excess activity of xanthine oxidase contributes significantly to vasodilatory impairment in patients with OSA.

The study was a sophisticated prospective, randomised, crossover design, minimising the presence of confounding variables and eliminating inherent individual variations in terms of the generation of free radicals, hyperaemic vascular reactivity or response to treatment. However, a number of arguable assumptions were made in the article of ELSOLH et al. [1].

First, endothelial function assessment using hyperaemiainduced flow-mediated vasodilation (FMD) is not always suitable for the assessment of endotheloial function in female obese patients with sleep apnoea. There is a significant relationship between FMD and brachial artery size; therefore, as males have larger arterial diameters, smaller FMD is noted in males [2]. Thus, the changes in FMD in males before and after therapeutic intervention are usually larger than those in females. As the current study did not examine the FMD results of females and males separately, the sex difference may exist in the study. Inversely, the FMD improvement after allopurinol treatment may be clearly indicated, when the males' results were analysed separately from the females' results.

Secondly, FMD of the brachial artery diminished with age [2]. Thus, the age distribution of the study sample affects the results of the FMD alterations after intervention. Because the authors examined subjects aged 29-60 yrs, this wide range of the population may not represent the genuine effects of allopirinol on the FMD in association with oxidative stress due to sleep apnoea itself.

Thirdly, obesity without sleep apnoea also causes endothelial dysfunction [3]. The FMD results should be standardised by the body mass index (BMI) or metabolic variables, when the FMD results are properly assessed. In the study by EL SOLH et al. [1], BMI ranged 23-67. We speculate that the FMD results in patients with a normal BMI of 24 might be very different from the extraordinarily obese patients with a BMI of 67 . It has also been reported that FMD is associated with systemic inflammation and glucose homeostasis in obese patients, independent of 
obesity [4]. Because both obesity and sleep apnoea cause systemic inflammation $[5,6]$, the association of the inflammatory markers and insulin sensitivity with FMD should be further examined.

Fourthly, the menopause and menstrual cycle significantly affect sleep apnoea and endothelial function [7, 8]. The menopausal transition is significantly associated with an increased likelihood of having sleep-disordered breathing, independent of known confounding factors [7]. FMD increases in menstrual phase, when serum oestradiol level is low and the value is comparable to that in male subjects [8]. Because endothelium-dependent vasodilatation varies during the menstrual cycle, the timing of FMD measurements of female subjects is critical for the precise assessment of allopinol effects.

The incidence of cardiovascular disease is lower in premenopausal females compared with males in the same age group; following menopause, the risk of mortality from cardiovascular disease increases in females [9]. FMD-induced vasodilatation is lower in females aged 55 yrs than those aged 35 yrs [10]. The lower FMD in females aged 55 yrs, compared with those aged $35 \mathrm{yrs}$, could be due to postmenopausal hormonal changes.

It has been suggested that endothelial function assessment using hyperaemia-induced FMD is adequately reproducible in healthy middle-aged males and females [2]. However, there are many confounding factors including age, sex, obesity, smoking, elevated blood lipids, high blood pressure and systemic inflammation. Thus, FMD measurement may not be an appropriate method for the assessment of the endothelial function in female obese patients with sleep apnoea.

Further study using a large sample size should be carefully assessed by age, obesity and sex differences. The improvement of endothelial function by allopurinol effects on the vascular function in patients with sleep apnoea will then be adequately realised.

\section{S. Teramoto*, H. Kume", Y. Yamaguchi, H. Yamamoto, Y. Hanaoka, M. Ishii, T. Ishii ${ }^{\oplus}$ and Y. Ouchi*}

Depts of *Geriatric Medicine and "Urology, Graduate School of Medicine, University of Tokyo, and "Dept of Respiratory Medicine, Nippon Medical School, Tokyo, Japan.

\section{REFERENCES}

1 El Solh AA, Saliba R, Bosinski T, Grant BJ, Berbary E, Miller N. Allopurinol improves endothelial function in sleep apnoea: a randomised controlled study. Eur Respir J 2006; 27: 997-1002.

2 Jensen-Urstad K, Rosfors S. A methodological study of arterial wall function using ultrasound technique. Clin Physiol 1997; 17: 557-567.

3 Shechter M, Beigel R, Freimark D, Matetzky S, Feinberg MS. Short-term sibutramine therapy is associated with weight loss and improved endothelial function in obese patients with coronary artery disease. Am J Cardiol 2006; 97: 1650-1653.
4 Williams IL, Chowienczyk PJ, Wheatcroft SB, et al. Endothelial function and weight loss in obese humans. Obes Surg 2005; 15: 1055-1060.

5 Teramoto S, Yamamoto H, Yamaguchi Y, Namba R, Ouchi Y. Obstructive sleep apnea causes systemic inflammation and metabolic syndrome. Chest 2005; 127: 1074-1075.

6 Teramoto S, Yamamoto H, Ouchi Y. Increased C-reactive protein and increased plasma interleukin- 6 may synergistically affect the progression of coronary atherosclerosis in obstructive sleep apnea syndrome. Circulation 2003; 107: E40.

7 Young T, Finn L, Austin D, Peterson A. Menopausal status and sleep-disordered breathing in the Wisconsin Sleep Cohort Study. Am J Respir Crit Care Med 2003; 167: 1181-1185.

8 Hashimoto M, Akishita M, Eto M, et al. Modulation of endothelium-dependent flow-mediated dilatation of the brachial artery by sex and menstrual cycle. Circulation 1995; 92: 3431-3435.

9 Jensen-Urstad K, Johansson J. Gender difference in agerelated changes in vascular function. J Intern Med 2001; 250: 29-36.

10 Perregaux D, Chaudhuri A, Mohanty P, et al. Effect of gender differences and estrogen replacement therapy on vascular reactivity. Metabolism 1999; 48: 227-232.

DOI: 10.1183/09031936.00104806

\section{From the authors:}

We thank S. Teramoto and colleagues for their comments on our recent article [1]. We agree that changes in flow-mediated vasodilation (FMD) are influenced by a variety of intrinsic and extrinsic factors such as endogenous, environmental and familial factors [2], of which age, sex and body mass index (BMI) are the classic examples. Indeed, analysis by sex of our data showed that male participants had larger arterial diameters and smaller FMD at baseline compared with female subjects $(6.1 \pm 2.6 \%$ in males and $8.0 \pm 1.7 \%$ in females $)$. However, in contrast to the remarks of $\mathrm{S}$. Teramoto and colleagues, the median FMD improvement after allopurinol treatment was larger in women $(4.3 \%$; $95 \%$ confidence interval (CI) $1.0-7.6 \%)$ than in their male counterparts $(3.4 \%$; $95 \% \mathrm{CI}$ $1.5-5.4 \%$; although not to a statistically significant degree. Correlation analyses also revealed no significant relationship between changes in FMD (before and after treatment) and either age $(r=0.2 ; p=0.5)$ or BMI $(r=0.06 ; p=0.85)$. We acknowledge that the power of the study is too small to detect any significant difference and we alluded to this limitation in the manuscript. As pointed out by S. Teramoto and colleagues, FMD is influenced by circulating levels of oestrogen and progesterone, and by the phase of the subject's menstrual cycle [3]. This variability would have been significant had our female participants been of a child-bearing age; however, only one of the four female subjects fell into that category. Finally, we concur with S. Teramoto and colleagues that a larger sample size would be needed to confirm our findings. Now that our randomised clinical trial has shown potential efficacy, we hope it stimulates further long-term research studies to determine the role and side-effects of allopurinol in the treatment of obstructive sleep apnoea-related endothelial dysfunction. 